\title{
Turbulence closure: turbulence, waves and the wave-turbulence transition - Part 1: Vanishing mean shear
}

\author{
H. Z. Baumert ${ }^{1}$ and H. Peters ${ }^{2}$ \\ ${ }^{1}$ Freie Universität, Dept. Mathematics and Computer Science, Berlin, and Institute for Applied Marine and Limnic Studies, \\ Hamburg, Germany \\ ${ }^{2}$ Earth and Space Research, Seattle, USA
}

Received: 12 September 2008 - Published in Ocean Sci. Discuss.: 14 November 2008

Revised: 20 January 2009 - Accepted: 10 February 2009 - Published: 6 March 2009

\begin{abstract}
This paper extends a turbulence closure-like model for stably stratified flows into a new dynamic domain in which turbulence is generated by internal gravity waves rather than mean shear. The model turbulent kinetic energy (TKE, $K$ ) balance, its first equation, incorporates a term for the energy transfer from internal waves to turbulence. This energy source is in addition to the traditional shear production. The second variable of the new two-equation model is the turbulent enstrophy $(\Omega)$. Compared to the traditional shear-only case, the $\Omega$-equation is modified to account for the effect of the waves on the turbulence time and space scales. This modification is based on the assumption of a non-zero constant flux Richardson number in the limit of vanishing mean shear when turbulence is produced exclusively by internal waves. This paper is part 1 of a continuing theoretical development. It accounts for mean shear- and internal wavedriven mixing only in the two limits of mean shear and no waves and waves but no mean shear, respectively.

The new model reproduces the wave-turbulence transition analyzed by D'Asaro and Lien (2000b). At small energy density $E$ of the internal wave field, the turbulent dissipation rate $(\varepsilon)$ scales like $\varepsilon \sim E^{2}$. This is what is observed in the deep sea. With increasing $E$, after the wave-turbulence transition has been passed, the scaling changes to $\varepsilon \sim E^{1}$. This is observed, for example, in the highly energetic tidal flow near a sill in Knight Inlet. The new model further exhibits a turbulent length scale proportional to the Ozmidov scale, as observed in the ocean, and predicts the ratio between the turbulent Thorpe and Ozmidov length scales well within the range observed in the ocean.
\end{abstract}

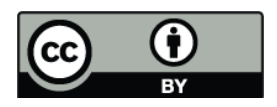

Correspondence to: H. Peters (hpeters@esr.org)

\section{Introduction}

\subsection{Motivation and goal}

Between strong turbulence in the surface and benthic boundary layers and weak and to some degree intermittent turbulence in the interior, the oceans harbor dynamically different regimes of turbulent flows. The turbulent mixing in these various regimes has been modeled in two distinctly different theoretical approaches with no commonalities in theory and little interaction between its proponents. On the one hand, mixing in boundary layers and mean shear flows, such as, for example, in tidal domains or in the Equatorial Undercurrent (EUC), is commonly represented by turbulence closure models. These models have their root in the turbulence theory of neutrally stratified flows and are completely ignorant of the presence of internal waves. On the other hand, mixing in the interior of the ocean is dominantly driven by internal gravity waves, and the prevailing model of this mixing has its root in nonlinear wave-wave interaction theory.

This paper describes a path toward reconciling the two different mixing theories in the sense of constructing a unified closure-like model that encompasses both mean shear- and wave-generated turbulence. Specifically, we present the first part of a continuing theoretical development. We only treat the two limits of (i) internal wave-driven mixing in the absence of mean shear and (ii) mean-shear driven mixing in the absence of wave-generated turbulence. A second paper on the simultaneous occurrence of wave- and mean sheargenerated mixing is in preparation.

It is our hope that a unified model of wave- and sheardriven mixing can improve the performance of circulation models. To characterize the current situation, we note that it has long been recognized that numerical circulation models of geophysical flows with embedded turbulence closures

Published by Copernicus Publications on behalf of the European Geosciences Union. 
have to be adjusted to account for wave-driven mixing in the interior of the ocean or atmosphere under study. This is commonly done by imposing a constant "background diffusivity." It seems typical rather than atypical that numerical simulations of Chesapeake Bay by Li et al. (2005) proved to be sensitive to the imposed background diffusivity and insensitive to details of the turbulence closure. A more sophisticated approach than a background diffusivity is clearly needed to account for turbulence in geophysical flows. This conclusion is also supported by an examination of a $K-\varepsilon$ closure applied to the permanently stratified and strongly sheared tidal flow of the Hudson River by Peters and Baumert (2007). They were able to reproduce strong mixing associated with strong shear but not weak mixing in weak shear.

This paper is motivated by one of the few publications which bridge the worlds of wave-driven and shear-driven turbulence models, D'Asaro and Lien (2000b), henceforth referred to as DL00. Examining the transition between internal waves and turbulence in Lagrangian drifter measurements, DL00 find some aspects of turbulence closures compatible with their results and encourage the development of new closures that are more completely attuned to their findings. This paper is an attempt to do so. The "wave-turbulence transition" described by DL00 provides the critical test for the merit of our ideas. We show below that our new model is constructed such that it exhibits the most important results of DL00.

\subsection{Basic dynamic considerations}

In order to make it easier for the reader to follow our approach, the following provides a brief summary of those aspects of traditional turbulence closure and oceanic internal wave-driven mixing on which our new development is based.

Ignoring convection, boundary forcing and surface wave breaking for the sake of simplicity, mean shear provides the dominant energy source of turbulence in boundary layers, energetic tidal and shear flows such as the EUC. This is the domain of turbulence closure, an approach with roots in nongeophysical hydrodynamics, engineering and in atmospheric and oceanic science, pioneered by Mellor and Yamada (1974, 1982) and Rodi (1987). Based on the Reynolds decomposition into mean and turbulent flow components, turbulence closure assumes that, possible buoyancy forcing aside, the mean shear provides the entire energy source of the turbulence. Specifically, the production of turbulent kinetic energy, $P$, varies with the square of mean shear, $P \sim S^{2}$. Internal wave forcing of turbulence or even the existence of waves is not considered.

Ignoring double diffusion for the sake of simplicity, mixing in the interior of the ocean is dominantly driven by internal inertia-gravity waves. Nonlinear wave-wave interaction theory has been invoked by McComas and Müller (1981) and Henyey et al. (1986) to model the energy flux through the internal wave spectrum to small wavelengths, and thus to tur- bulence. The energy flux $\tilde{P}$ to short waves approximately equals the energy flux through the turbulence cascade and the turbulent dissipation rate $\varepsilon$.

Gregg (1989) (henceforth G89; see also Polzin et al., 1995) combined the internal wave interaction theories with oceanic dissipation measurements and established the relationship $\tilde{P} \sim \varepsilon$ quantitatively with $\tilde{P} \sim E^{2}$, where $E$ is the energy density of the wave field. Following G89 this relationship corresponds to $\tilde{P} \sim\left\langle S_{10}^{4}\right\rangle: \tilde{P}$, and hence $\varepsilon$, varies with the fourth power of the RMS internal wave shear at a vertical scale of $10 \mathrm{~m}, S_{10}$. The difference in the scaling of the mean shear turbulent kinetic energy (TKE) production $P \sim S^{2}$ and the wave production term $\tilde{P} \sim\left\langle S_{10}^{4}\right\rangle$ is fundamental to our development.

The following text outlines the flow physics behind the key term $\tilde{P}$. The oceanic internal wave field occupies a broadband frequency-wavenumber spectrum which is astonishingly similar throughout the deep sea. This observation was synthesized in the Garrett and Munk (GM; 1972, 1975) oceanic internal wave model. The energy flux $\tilde{P}$ to small scales, $\tilde{P}$, is funneled through a "saturated" or "compliant" part of the vertical wavenumber shear spectrum of the wave field with $m^{-1}$ vertical wavenumber $(m)$ dependence and constant spectral "level." This level is invariant under variations of the energy density of the internal wave field $(E)$ and $\tilde{P}$ such that we refer to such wave fields with $\tilde{P}>0$ as "saturated."

As shown, e.g., by Gregg et al. (1993) and consistent with the GM model, the overall vertical shear spectrum in the interior ocean is approximately flat to a cyclic vertical wavenumber $m$ of about $0.1 \mathrm{~m}^{-1}$. The already mentioned saturated wavenumber range begins at $m>0.1 \mathrm{~m}^{-1}$, a phenomenon similarly observed in the atmosphere (e.g., Fritts, 1989; Fritts and Alexander, 2003). The high- $m$ end of the of the saturated band transitions into the turbulent inertial subrange. A sketch of this vertical wavenumber spectrum of shear is provided in Fig. 1. It is approximately related to the vertical energy spectrum by a multiplication by $(2 \pi m)^{2}$. The energy spectrum is too "red" to readily reveal its properties to the eye.

The nature of being saturated is what distinguishes the broadband oceanic internal wave field from internal waves in stably stratified laboratory flows. The laboratory necessarily restricts the range of space and time scales of internal wave fields and thus severely limits the range of possible wavewave interactions. Consequently, $\tilde{P}$ in a laboratory setup is likely zero or insignificant. An example is the turbulence decay experiment of Dickey and Mellor (1980), in which the kinetic energy at first decays fast, $\sim t^{-1}$, as a consequence of nonlinear turbulent interactions and later much more slowly in a molecular viscous decay of non-turbulent, wave-like and possibly vortical motions.

We refer to internal waves with the property $\tilde{P}=0$ as "unsaturated." In the analyzes of Baumert and Peters (2004) (henceforth BP04) and in Baumert and Peters (2005) the 
transfer of energy from TKE to unsaturated waves, $W$ in the following, proved to be important for making closures consistent with the turbulent length scales observed in the laboratory. These papers did not consider waves as a source of TKE. In some laboratory experiments examined in Baumert and Peters (2000) (henceforth BP00) and in BP04, turbulence provides the energy source for weak internal waves. In contrast, the strong internal wave field of the ocean is dominantly powered by the surface wind stress generating near-inertial motions in the surface mixed layer and by interactions of the barotropic tide with variable topography. The internal wave energy propagates into and fills the world ocean where it is transferred to turbulence and dissipated.

At this point we can more explicitly summarize the wave-turbulence transition in D'Asaro and Lien (2000b) (or DL00). At low internal wave energy density $E$, the spectral energy flux to turbulence, $\tilde{P}$, scales with $E^{2}$ as in the G89 model, $\tilde{P} \sim \varepsilon \sim E^{2}$. Above a level of $E$ indicating the actual "wave-turbulence transition", this relationship changes to $\tilde{P} \sim E^{1}$. Our model outlined below replicates this transition.

1.3 Our take on turbulence closure and its extension toward wave-driven mixing

Finally, we need to briefly address the idiosyncrasies of our past and present handling of turbulence closures. We are working with a $K-\Omega$ equation system based on previous papers, Baumert and Peters $(2000,2004,2005)$ and Baumert (2005a), henceforth referred to as BP00, BP04, BP05 and $\mathrm{B} 05 \mathrm{a}$, respectively. Our primary turbulence variables are the turbulent kinetic energy (TKE, $K$ ) and the enstrophy $(\Omega)$ of the turbulence, the latter having the dimension of time ${ }^{-1}$. The $\Omega$-equation sets the space and time scales of the turbulence, more specifically, of the energetic eddies.

In the above-mentioned series of studies our emphasis has been on the proper growth, decay and steady state behavior for unbounded shear flows in constant mean shear and constant stratification far from solid boundaries, and under laboratory conditions. For this limit BP00 show that two-equation closures using different variables in the length scale-related equation, e.g., $\Omega, \varepsilon$ or $K, L$ as in the Mellor and Yamada (1982) closure, are mappable onto each other and differ only in the coefficients of that equation. In this limit, there is no inherent advantage of one variable over the others. Among the many choices of a second state variable, $\Omega$ is best in our view because $\Omega$ stays in close analogy to the RMS vorticity as discussed by Wilcox (1998), whereby, under certain circumstances, vorticity is a conserved variable in the Euler equations. Only our form of the $K-\Omega$ model reproduces the law of the wall with von Kármán constant equal to $0.399 \approx 0.4$, which is the international standard value.

The turbulence model described herein extends into the domain of internal wave-driven mixing. Hence the TKE balance has to incorporate the internal wave spectral energy flux

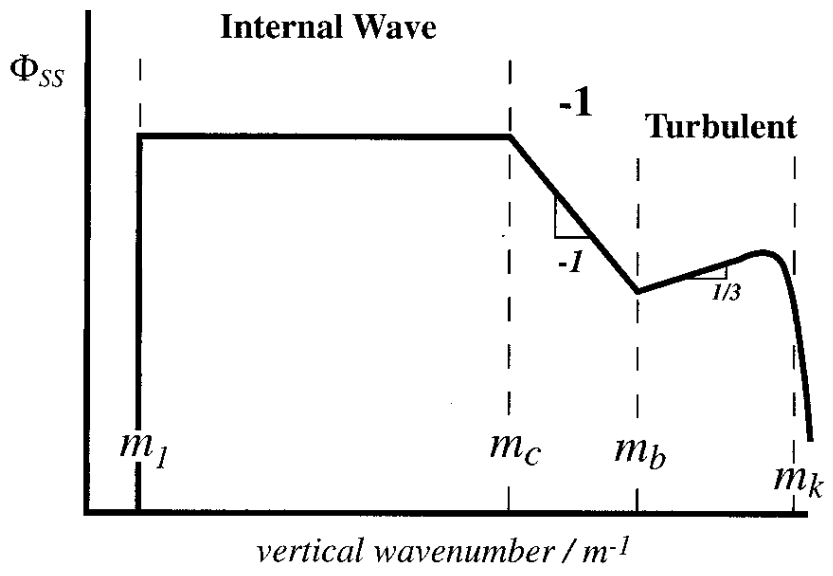

Fig. 1. Features of Eulerian oceanic power spectra of the vertical shear of the horizontal velocity, $\Phi_{S S}$, as a function of the vertical wavenumber $m$ after D'Asaro and Lien (2000; their Fig. 3), log$\log$ plot. The GM-like internal wave band ranges from $m_{1}$ to $m_{c}$, followed by the saturated band with $m^{-1}$ behavior and invariant level. The turbulent inertial subrange and the final viscous drop off reside between $m_{b}$ and $m_{k}$, the latter being the inverse of the Kolmogorov scale.

$\tilde{P}$ as an extra energy source term. The nature of TKE production by mean shear of $P \sim S^{2}$ and Gregg's (1989) $\tilde{P} \sim\left\langle S_{10}^{4}\right\rangle$ differ severely. And thus an internal wave-related energy source can not simply be incorporated into the shear production term in the TKE equation (as done, e.g., by Canuto et al., 2001a). A further motivation for keeping $\tilde{P}$ as a separate source term in the $K$-equation is the perspective of integrating an internal wave model providing $\tilde{P}$ in parallel with mean flow and closure equations.

After the comparatively simple and straightforward step of adding $\tilde{P}$ to the TKE equation, we also have to account for the effect of internal waves on the space and time scales of the turbulence, which means modifying the $\Omega$ equation. There is no previous guidance for this non-trivial step. Rather than making assumptions directly for the space or time scale, we base our modification of the enstrophy balance on an assumption about the efficiency of mixing. We assume that, as the mean shear $S$ becomes small and the mean-flow gradient Richardson number $\left(R_{g}=N^{2} / S^{2}\right)$ becomes large, the flux Richardson number $R_{f}$ approaches a constant, non-zero value, an invariant of our model. The assumption that $\lim _{R_{g} \rightarrow \infty}\left(R_{f}\right)=R_{f}^{\infty}=$ constant is based on oceanic observations of the misnamed "mixing efficiency" $\Gamma=R_{f} /\left(1-R_{f}\right)$ in low mean-shear environments (Osborn, 1980; Oakey, 1982; Moum, 1990, 1996a). Constant $R_{f}^{\infty}$ already appeared in an empirically motivated turbulence model of Schumann and Gerz (1995) and was used in the validation of a conventional $K-\varepsilon$ closure by Peters and Baumert (2007).

The theoretical analysis below shows that our assumption of a specific constant $R_{f}^{\infty}$ at $R_{g} \rightarrow \infty$ is sufficient to close the 
system of $K-\Omega$ equations with respect to the internal waves and to ensure the uniqueness of its physically relevant solutions.

\subsection{Outline of the development}

This paper is organized as follows. A brief discussion of turbulence regimes as function of the gradient Richardson number continues this introduction and further characterizes our approach (Sect. 2). Therein we first review the cases of neutral stratification (Sect. 3) and stable stratification without internal wave-driven turbulence (Sect. 3.1). Thereafter we switch to internal waves with a summary of the G89 model of open ocean internal wave-induced turbulent dissipation (Sect. 3.2). As our model is to be compatible with laboratory as well as oceanic conditions, we need to address nonlinear and viscous energy losses of internal waves, too (Sect. 3.3). Only then we can present the TKE balance in the presence of waves and mean shear (Sect. 4).

Thereafter we limit the discussion to the case of vanishing mean shear and explain how the assumption of a constant, non-zero flux Richardson number leads to a modification of the $\Omega$-equation consistent with the wave-dominated dynamics (Sect. 5). We then show that the just-constructed model is consistent with the wave-turbulence transition as analyzed by D'Asaro and Lien (2000b) (Sect. 6). That is, we discuss the behavior of our two-equation model when the energy density of the internal waves increases from conditions of small $E$ observed in the deep sea to large $E$ observed, for example, in tidal flows. The paper concludes with a summary and a brief discussion.

\section{Turbulence regimes}

The mean-flow gradient Richardson number,

$R_{g}=N^{2} / S^{2}$,

the ratio of the buoyancy frequency $N$ and the vertical shear of the mean horizontal velocity $S$ is a most fundamental and useful characteristic of stratified flows. Different ranges of $R_{g}$ correspond to different hydrodynamic regimes without a saturated internal wave field. In stratified, spatially homogeneous shear layers in laboratory experiments, direct numerical simulations and idealized theoretical considerations the following ranges are found.

(a) $R_{g} \leq R_{g}^{a}=0$ : unstable and neutral stratification, convective and neutral turbulence, no internal waves.

(b) $R_{g}^{a}<R_{g}<R_{g}^{b}=1 / 4$ : neutral and stable stratification, shear-dominated growing turbulence, coexistence of turbulence and nonlinear internal waves.

(c) $R_{g}^{b}<R_{g}<R_{g}^{c}=1 / 2$ : stable stratification, wavedominated decaying turbulence, coexistence of turbulence and internal waves. (d) $R_{g}^{c}<R_{g}$ : stable stratification, waves-only regime.

The usefulness of the concept of homogeneous shear layers is discussed in BP00 and BP04. They are approximated in the shear flow experiments of the Van Atta group (e.g., Rohr et al., 1988). The numerical values for $R_{g}^{a}, R_{g}^{b}$ and $R_{g}^{c}$ given above hold only for the asymptotic case of an infinite Reynolds number $(R e)$. Finite $R e$ result in $R_{g}^{b}=0.12$ to 0.25 in the laboratory (Tjernström, 1993; Shih et al., 2000) and in the atmosphere (see the review of Foken, 2006). In this concept the flow conditions are simple and controlled so that the analyzes of Richardson (1922), Miles (1961), Howard (1961) and Abarbanel et al. (1984) concerning flow instability and the existence of turbulence are fully applicable. The preceding reflects "laboratory flow physics."

Most of the ocean and atmosphere deviate qualitatively from the preceding classification of flow regimes as turbulence occurs even at very large $R_{g}$ as a consequence of the presence of saturated internal waves as already noted above. Traditionally, turbulence closures have included a "critical" Richardson number above which turbulence is suppressed. This corresponds to laboratory conditions as classified above. For example in the closures of Mellor and Yamada (1982) and Canuto et al. (2001a), this suppression is implemented through the stability functions of the respective closures with $R_{g}^{c} \approx 0.2$ in variants of the Mellor and Yamada (1982) closure and $R_{g}^{c}$ in the range of 0.8 to 1 in the case of Canuto et al. (2001a).

Recent attempts to ameliorate these fundamental problems do not seek the primary reason in the missing energy flux from the saturated internal wave field into the TKE pool. They are looking for other reasons. For stratified boundary layers, which clearly differ from homogeneous layers to which the critical $R_{g}$ concept exclusively applies, a nongradient correction of the traditional buoyancy flux formulation has been applied by Zilitinkevich et al. (2007), which leads to many new parameters. Sukoriansky et al. (2006), Sukoriansky (2007) and Galperin et al. (2007) use a construct of an advanced spectral model coupled with an algebraic length-scale prescription. Thus the non-trivial criticality problem, where space and time scales exhibit non-trivial behavior, is covered by their choice of the non-physical length-scale relationship.

We follow a different path; we focus on the dynamics of turbulent flows as symbolized by our introduction of the TKE source term $\tilde{P}$ as a term of leading order. It is important to realize that variations in $\tilde{P}$ are not related to mean shear $S$. They are related to variations of the internal wave RMS shear, $\tilde{S}_{10}$, in the form $\tilde{P} \sim\left\langle S_{10}^{4}\right\rangle$ as shown in G89. Below, we outline a turbulence model that follows the above $R_{g}$-based characterization of flow regimes in the absence of saturated internal waves while allowing for steady state turbulence at any $R_{g}>0$ in the presence of saturated waves. In steady conditions without saturated internal waves, the flow laminarizes above $R_{g}^{c}$, and the critical Richardson number retains 
its meaning from the classical analyzes (Richardson, 1922; Miles, 1961; Howard, 1961; Abarbanel et al., 1984). The presence of a saturated internal wave field in oceans and the atmosphere is a first-order process which modifies the classical view of flow instability but does not make it obsolete.

\section{Neutral stratification $\left(R_{g}=0\right)$}

In order to make our turbulence model transparent we first discuss its most important properties in the simple case of neutral stratification in this section and for stable stratification in the absence of saturated waves in the following section. This is the essence of BP04 but formulated in $K-\Omega$ format following B05a. For a horizontally homogeneous flow with vanishing vertical mean-flow component, the governing equations are

$$
\begin{aligned}
& \frac{\partial K}{\partial t}-\frac{\partial}{\partial z}\left(v_{t} \frac{\partial K}{\partial z}\right)=v_{t}\left(S^{2}-\Omega^{2}\right) \quad \text { and } \\
& \frac{\partial \Omega}{\partial t}-\frac{\partial}{\partial z}\left(v_{t} \frac{\partial \Omega}{\partial z}\right)=\frac{1}{\pi}\left(\frac{S^{2}}{2}-\Omega^{2}\right)
\end{aligned}
$$

where $v_{t}$ is the eddy viscosity, given by the PrandtlKolmogorov relationships,

$v_{t}=\frac{K / \pi}{\Omega}=\frac{(K / \pi)^{2}}{\varepsilon}$.

$S^{2}$ is square of mean vertical shear and

$\varepsilon=\pi^{-1} \Omega K=v_{t} \Omega^{2}$

is the turbulent dissipation rate.

Equations (2) and (3) follow the preference of BP04 and B05a to avoid empirical parameters where possible and to express them as integers, rational numbers or mathematical entities, such as, e.g., $\pi$. The diffusion terms on the left-hand side of closure equations such as Eqs. (2) and (3) carry extra parameters in many closures. The parameters of Eq. (3) can be made explicit by writing it as

$\frac{\partial \Omega}{\partial t}-\frac{1}{\sigma_{\Omega}} \frac{\partial}{\partial z}\left(v_{t} \frac{\partial \Omega}{\partial z}\right)=\left(c_{1} S^{2}-c_{2} \Omega^{2}\right)$.

The choices of BP04 and B05a are $\sigma_{\Omega}=1, c_{1}=(2 \pi)^{-1}$ and $c_{2}=\pi^{-1}$.

The integral length and time scales of the energycontaining turbulent eddies, $L$ and $\tau$, respectively, can be expressed in terms of $K$ and $\Omega$ as follows:

$L=\frac{(K / \pi)^{3 / 2}}{\varepsilon}=\frac{\sqrt{K / \pi}}{\Omega}$,

$\tau=2 \frac{K}{\varepsilon}=\frac{2 \pi}{\Omega}$.

The Eqs. (2, 3, 4, and 5) differ only slightly from the traditional and well-accepted $K-\omega$ equations by Wilcox (1998).
3.1 Stable stratification, laboratory $\left(0 \leq R_{g}<1 / 2\right)$

In layers far from the bottom or surface boundary the vertical diffusion terms in Eqs. (2 and 3) can be neglected. For this case BP04 write the TKE balance as

$$
\begin{aligned}
\frac{d K}{d t} & =-\left\langle w^{\prime} u^{\prime}\right\rangle S-W-\frac{1}{\rho} g\left\langle w^{\prime} \rho^{\prime}\right\rangle-\varepsilon . \\
& =P-\mathcal{B}-\varepsilon,
\end{aligned}
$$

Here,

$P=v_{t} S^{2}$

is the shear production of TKE, and

$\mathcal{B}=B+W=2 v_{t} N^{2}$

is the total buoyancy-related loss rate of TKE, which does not directly depend on shear or Richardson number. $W$ is the energy transfer from TKE to internal waves, and $B$ is the common buoyancy flux,

$B=\mu_{t} N^{2}=-\mu_{t} \frac{g}{\rho_{0}} \frac{d \bar{\rho}}{d x_{3}}$.

The eddy diffusivity $\mu_{t}$ is related to $v_{t}$ through the turbulent Prandtl number function $\sigma$,

$\mu_{t}=v_{t} / \sigma$.

Within BP04 we developed a generalized form of the turbulent Prandtl number $\sigma$ as a function of the frequency ratio $N / \Omega$,

$\sigma=1 / 2\left(1-N^{2} / \Omega^{2}\right)^{-1 / 2}$.

Even with stable stratification the enstrophy Eq. (3) remains unchanged from the case of $N^{2}=0$ as it does not contain a buoyancy-related term.

A state of structural equilibrium introduced in BP00 for homogeneous shear layers corresponds to exponential growth, decay or steady state of TKE. BP04 show that structural equilibrium corresponds to $d \Omega / d t=0$. With this, Eq. (3) is converted to

$\Omega^{2}=1 / 2 S^{2}$

such that Eq. (15) becomes

$\sigma=1 / 2\left(1-2 R_{g}\right)^{-1}$.

For structural equilibrium some algebra of Eqs. (1), (5, 10, and 12) results in

$\frac{d K}{d t}=2 v_{t} S^{2}\left(1 / 4-R_{g}\right)$.

Equations (17 and 18) exhibit the properties discussed already in Sect. 2. Steady state occurs at $R_{g}=1 / 4$, growing turbulence at $R_{g}<1 / 4$, decaying turbulence for $R_{g}>1 / 4$. Further, Eq. (17) shows that turbulence cannot exist at all for 
$R_{g}>1 / 2$. At the threshold $R_{g}=1 / 2$ TKE is converted into wave energy (term $W$ ). These results are well supported by various laboratory measurements as discussed in BP00, $\mathrm{BP} 04$ and BP05. For the proper interpretation of the preceding statement of "no waves at $R_{g}>1 / 2$ " the reader is reminded that this section addresses laboratory conditions in which there are no saturated waves.

We close this chapter by recalling an important result of BP04 which is used later below:

$L / L_{O}=\left(2 R_{g}\right)^{3 / 4}$,

where $L_{O}=\varepsilon^{1 / 2} N^{-3 / 2}$ is the Ozmidov scale. Equation (19) is valid in the case of structural equilibrium.

\subsection{Gregg (1989): internal waves and dissipation}

In preparation for introducing $\tilde{P}$ into our turbulence model we now review the Gregg (1989) model of internal wavedriven mixing. G89 assembled a range of dissipation measurements from various locations in the ocean with simultaneous measurements of the vertical shear of the horizontal velocity, $S_{10}$. All these observations were taken with vertical profilers. Shear was evaluated by integrating shear spectra to $m=1 / 10 \mathrm{~m}$, that is by integrating the flat internal wave part of the spectrum shown in Fig. 1 to $m_{c}$, to the beginning of the saturated range. The nonlinear internal wave interaction theories of McComas and Müller (1981) and Henyey et al. (1986) relate $E$ to $\tilde{P}$ as

$\tilde{P} \sim N^{-2} E^{2}$.

Noting that $E$ WKB-scales with $N^{2}$ and invoking the GM model following G89, Eq. (20) translates to

$\tilde{P} \sim N^{2}\left\langle S_{10}^{4}\right\rangle$.

The shear term has to be understood as an ensemble average with the property $\left\langle S_{10}^{4}\right\rangle=2\left\langle S_{10}^{2}\right\rangle^{2}$ (G89).

G89 assumed that the observed $\varepsilon$ approximately equals $\tilde{P}$,

$\tilde{P}=\varepsilon+B \approx \varepsilon$,

noting that for negligible mean flow shear, $S^{2} \ll\left\langle S_{10}^{2}\right\rangle$, the buoyancy flux produced by the wave-driven mixing amounts to only about $20 \%$ of $\varepsilon$ (Oakey, 1982). G89 finds the waveinduced dissipation $\tilde{\varepsilon}$ as

$\tilde{\varepsilon}=a_{1} \frac{\left\langle N^{2}\right\rangle}{N_{0}^{2}} \frac{\left\langle S_{10}^{4}\right\rangle}{S_{G M}^{4}}$,

where angle brackets indicate ensemble averages as before. The constant $a_{1}$ specifies the dissipation rate induced by an internal wave field at the GM energy level and $N=N_{0}, a_{1}=7 \times 10^{-10} \mathrm{~m}^{2} \mathrm{~s}^{-3} . N_{0}=5.2 \times 10^{-3} \mathrm{~s}^{-1}$ is the buoyancy frequency used in the WKB scaling in GM, and $S_{G M}^{2}=1.96 \times 10^{-5} \mathrm{~s}^{-2}\left\langle N^{2}\right\rangle / N_{0}^{2}$ is the squared shear from GM. Combining $N_{0}$ and $S_{G M}$ for $N=N_{0}$ into a RMS wave Richardson number leads to $R_{g}^{G M}=\left\langle N^{2}\right\rangle /\left\langle S_{G M}^{2}\right\rangle \approx 1.4$.

\subsection{Internal Wave Energy Balance}

The following is a balance equation for $E$ inspired by G89 but tailored to our concept by adding a term related to the viscous dissipation of wave energy.

$$
\begin{aligned}
\frac{d E}{d t} & =X-c_{1} E-c_{2} E^{2} \\
& =\Pi+W-E / \mathcal{T}, \\
\mathcal{T} & =\left(c_{1}+c_{2} E\right)^{-1} \\
X & =\Pi+W
\end{aligned}
$$

Here, $X$ is the energy flux into the internal wave field from external sources. It consists of two parts: $\Pi$ stands for the energy input from wind and tides at low frequencies, and $W$ is the energy flux from TKE to waves introduced in BP04 and already mentioned above. $\mathcal{T}$ is the relaxation time constant of the wave field. There are two damping terms, $c_{1} E$ and $c_{2} E^{2}$. The first, with $c_{1} \propto v m^{2}$, describes the molecular frictional damping of wavenumber $m$ by molecular kinematic viscosity $v$. A wave with $10 \mathrm{~m}$ vertical wavelength, for example, has a long molecular life time of about one month. The second term, $c_{2} E^{2}$, is related to the nonlinear energy transfers to large $m, \tilde{P}$. Based on many observations, G89 report that $c_{2} \approx 6.4 \mathrm{~d}^{-1}[E]^{-1}=7.4 \times 10^{-5} \mathrm{~m}^{-2} \mathrm{~s}^{1}$. Here $[E]$ stands for the units of $E$, which are $\mathrm{m}^{2} \mathrm{~s}^{-2}$ in the SI system.

With respect to the TKE production by internal wave shear and breaking,

$\tilde{P}=c_{2} E^{2}$,

we introduce an internal wave saturation index $f_{s}$ through

$f_{s}=\frac{\tilde{P}}{X}=c_{2} \frac{E^{2}}{X}$

with the property

$0 \leq f_{s} \leq 1$.

In steady state and for large energy input $X, E$ is sufficiently large so that the linear term in Eq. (24) can be neglected. Then

$\tilde{P} \approx X \approx c_{2} E^{2} \approx \Pi+W$,

and hence $f_{s} \approx 1$.

G89 and our treatment correspond to the properties of GM-like open ocean internal waves. Wave fields with different spectra and a different spectral energy flux, such as those on the continental shelf analyzed by MacKinnon and Gregg (2003b), are beyond the scope of our turbulence model.

\section{TKE balance with waves}

We now recall the TKE balance Eqs. (10 and 12), add $\tilde{P}$ using Eqs. $(27,28,29)$ and obtain

$$
\begin{aligned}
\frac{d K}{d t} & =P+\sim P-(B+W)-\varepsilon \\
& =P+f_{s}(\Pi+W)-(B+W)-\varepsilon \\
& =P+f_{s} \Pi+\left(f_{s}-1\right) W-B-\varepsilon .
\end{aligned}
$$


To perform practical calculations with Eq. (32) it is necessary to use the dynamic equation for $E$, Eqs. (24) and (29) to compute $f_{s}$, or to at least have an estimate of $f_{s}$.

In the laboratory there typically are no external energy sources for internal waves, $\Pi=0$. Observation times are usually short and the spatial extent of experiments is limited such that saturated waves cannot form. Hence, according to Eqs. (25) and (29), we expect $f_{s} \approx 0$. In the ocean we expect strong internal wave forcing, $\Pi>0$. There is no time limit preventing the establishment of a saturated wave field, and thus we expect $f_{s} \rightarrow 1$.

The case $f_{s}=0$ transforms Eq. (32) back into the form Eq. (12), which expresses laboratory flow conditions. The case $f_{s}=1$ leads to

$$
\frac{d K}{d t}=P+\Pi-B-\varepsilon,
$$

where the term $W$ no longer appears. In mathematical terms the steady state of purely wave-generated turbulence is given by $d K / d t=0$ and $P=0$, such that

$\Pi=B+\varepsilon$.

The external energy forcing of the internal wave field is transferred spectrally to the turbulence and is balanced by the turbulent buoyancy flux and dissipation rate.

\section{Enstrophy balance with waves}

Above, we have modified the TKE balance by adding an extra energy source term. This is a straightforward procedure as such energy source terms are additive quantities. Modifying the enstrophy balance to account for the effects of saturated internal waves is less obvious. This task is done herein only for the case of vanishing mean shear, while the general case is the subject of a future publication.

To begin with, we show that the enstrophy balance does indeed have to be changed in the presence of waves. For vanishing mean-flow shear, $S=0$, the state of structural equilibrium of Eq. (3), i.e. the case $d \Omega / d t=0$, has only the trivial solution $\Omega=0$. In order to avoid degenerate and non-physical solutions like this, we modify Eq. (3) formally as follows,

$\frac{\partial \Omega}{\partial t}-\frac{\partial}{\partial z}\left(v_{t} \frac{\partial \Omega}{\partial z}\right)=\frac{1}{\pi}\left(\tilde{\Omega}^{2}-\Omega^{2}\right)$,

where $\tilde{\Omega}$ is an unknown which we have to determine. ${ }^{1}$.

Considering the role of shear in the $\Omega$ balance one might speculate that $\tilde{\Omega}^{2}$ should be replaced by the RMS wave shear, say, by $S_{10}$. But that turned out to be wrong as it neglects higher-order contributions of the nonlinear wave field to the

\footnotetext{
${ }^{1}$ In the general case of non-zero mean shear, $\tilde{\Omega}$ is required to abide by the limiting condition $\lim _{\Pi \rightarrow 0} \tilde{\Omega}(\Pi, S)=\sqrt{S^{2} / 2}$ so that the case of the absence of saturated waves, $\tilde{P}=0$, can be recovered.
}

enstrophy balance of turbulence. These contributions are difficult to quantify in consideration of McComas and Müller (1981) and Henyey et al. (1986). We therefore proceeded along a path as follows. We determine $\tilde{\Omega}$ for $\tilde{P}>0, S=0$, $N^{2}>0$ by invoking the mixing efficiency $\Gamma$ as noted in the introduction to this paper. $\Gamma$ is defined as

$\Gamma=\frac{B}{\varepsilon}$.

$\Gamma$ was first estimated from microstructure measurements by Oakey (1982) with a result of $\Gamma \approx 0.2$ amid scatter and with substantial systematic uncertainty. A constant value of 0.2 has since been used in many publications. It is important to note that $\Gamma=$ constant can hold at most for large $R_{g}$ at $N^{2}>0$, that is in wave-dominated mixing. More generally, $N^{2} \rightarrow 0$, and thus $R_{g} \rightarrow 0$, imply $B \rightarrow 0$ and hence $\Gamma \rightarrow 0$. Systematic variations of $\Gamma$ have been associated with the age of turbulent overturns by Wijesekera and Dillon (1997). In assuming $\Gamma=\tilde{\Gamma}=0.2$ in wave-driven mixing herein, we implicitly assume that we are averaging over an ensemble of mixing events with similar evolution in a uniform wave field as expressed in the GM model.

We now rewrite Eq. (36) for the waves-only case using Eq. (13, 14, and 15) as follows:

$$
\begin{aligned}
B & =\tilde{\Gamma} \varepsilon=\mu_{t} N^{2}=\frac{v_{t}}{\sigma} N^{2} \\
& =2 v_{t}\left(1-\frac{N^{2}}{\tilde{\Omega}^{2}}\right) N^{2} .
\end{aligned}
$$

Based on Eqs. (5) and (4), we replace $\varepsilon$ as $\varepsilon=\tilde{\Omega} K / \pi$ and $v_{t}$ as $v_{t}=\tilde{K} /(\pi \tilde{\Omega})$. After a little algebra we find

$$
\tilde{\Gamma} \frac{\tilde{\Omega}^{2}}{N^{2}}=2\left(1-\frac{N^{2}}{\tilde{\Omega}^{2}}\right) .
$$

With the abbreviation $\eta=\tilde{\Omega} / N$ this relationship is equivalent to

$\tilde{\Gamma} \eta^{4}-2 \eta^{2}+2=0$.

This equation has four solutions. Two of them are negative and can thus immediately be excluded. The smaller of the two positive solutions is the physically correct one. For $\tilde{\Gamma}=0.2$ it is given by

$\eta=\sqrt{(1 \pm \sqrt{1-2 \tilde{\Gamma}}) / \tilde{\Gamma}}=1.06$.

For asymptotically small mean shear this value of $\eta$ leads to values of the $\Omega$-to- $N$ ratio, the $L$-to- $L_{O}$ ratio and the turbulent Prandtl number, given below in Eqs. (42-44). Along the way we invoke Eq. (7) to obtain the turbulent length scale $L$, and Eq. (5) to eliminate $K$ in favor of $\varepsilon$ and the Ozmidov 
scale, $L_{O}=\varepsilon^{1 / 2} N^{-3 / 2}$.

$$
\begin{aligned}
\tilde{\Omega} / N & =1.06>1, \\
L / L_{O} & =0.91<1, \\
\sigma & =\frac{1 / 2}{1-N^{2} / \tilde{\Omega}^{2}}=4.44 .
\end{aligned}
$$

Hence, the turbulent enstrophy of the waves-only case approximately equals the buoyancy frequency, staying just above it in the turbulent, rather than wave domain, and the turbulent length scale approximately equals the Ozmidov scale. It is well known that the Ozmidov scale approximately equals turbulent overturning scales in the low-mean shear open ocean thermocline. Results by Crawford (1986) can be reinterpreted as $L_{t h} / L_{O}=1.5 \pm 0.4$. These confidence bounds include the result of Dillon (1982), $L_{t h} / L_{O}=1.25$. Based on laboratory data BP04 link $L_{t h}$ to the model $L$ as $L_{t h}=2 L$. With this, our model indicates $L_{t h} / L_{O} \approx 1.8$, which is well-compatible with the oceanic observations.

Above, the generalized Prandtl number $\sigma$ is given only for reasons of completeness. Due to the absence of shear it does not describe the ratio between momentum and scalar fluxes and may be characterized as wave-degenerate.

\section{The wave-turbulence transition}

\subsection{Turbulent and wave energy, and the WT transition in DL00}

D'Asaro and Lien (2000b) analyze observations of internal waves and turbulence in regimes of varying total energy density from the low-energy open ocean thermocline to highly energetic flows in fjords. They examine velocity spectra and turbulent dissipation as a function of energy levels varying from below to above the "wave-turbulence (WT) transition". After a summary of DL00, further below, we show how our model replicates the WT transition.

The core of D'Asaro and Lien (2000b) is based on measurements of the vertical velocity $(w)$ with Lagrangian drifters (D'Asaro and Lien, 2000a). Lagrangian frequency spectra of $w, \Phi_{w}$, extend from wave motions to turbulent motions as shown schematically in Fig. 2. Following DL00, waves reside at frequencies $f \leq \omega \leq N$, where $f$ is the Coriolis parameter and $\omega$ is frequency. The turbulence resides at $\omega \geq N$. As the level of $\Phi_{w}$ increases in the internal wave band, so does its level in the turbulent band. Fig. 2 further sketches out that $\Phi_{w}$ drops by $\Delta_{W T}$ at $\omega=N$ upon the transition from the wave regime into the turbulence regime. With increasing level of $\Phi_{w}, \Delta_{W T}$ becomes smaller, $\Delta_{W T}=0$ indicating that the wave-turbulence transition threshold has been reached. With further increase of the spectral energy level $\Delta_{W T}$ remains 0 and turbulence now dominates the total vertical velocity variance.
With reference to Fig. 2, we define the wave-, turbulent and total vertical velocity variances as

$$
\begin{aligned}
\left\langle\tilde{w}^{2}\right\rangle & =\int_{f}^{N} \Phi_{w}(\omega) d \omega, \\
\left\langle w^{\prime 2}\right\rangle & =\int_{N}^{\infty} \Phi_{w}(\omega) d \omega, \\
\left\langle w^{2}\right\rangle & =\left\langle\tilde{w}^{2}\right\rangle+\left\langle w^{\prime 2}\right\rangle .
\end{aligned}
$$

Angled brackets indicate ensemble averages as before. In this notation the changing composition of the total energy between waves and turbulence is expressed as $\left\langle\tilde{w}^{2}\right\rangle>\left\langle w^{\prime 2}\right\rangle$ below the WT transition and $\left\langle\tilde{w}^{2}\right\rangle<\left\langle w^{\prime 2}\right\rangle$ above.

Perhaps more important than the changing composition of $\left\langle w^{2}\right\rangle$ into its turbulent and wave parts is the corresponding change in the relationship between total energy and the spectral energy flux $\tilde{P}$. DL00 show that, at small $\left\langle w^{2}\right\rangle, \tilde{P}$ scales with the second power of $\left\langle w^{2}\right\rangle, \tilde{P} \sim\left\langle w^{2}\right\rangle^{2}$. At large $\left\langle w^{2}\right\rangle$ above the WT transition, $\tilde{P}$ scales with the first power of $\left\langle w^{2}\right\rangle, \tilde{P} \sim\left\langle w^{2}\right\rangle^{1}$. We repeat that increasing wave energy $E$, and thus increasing $\left\langle w^{2}\right\rangle$ according to Fig. 2, implies increasing spectral energy flux $\tilde{P}$ according to Eq. (28) and thus increasing $\varepsilon$.

The vertical velocity variance $\left\langle w^{2}\right\rangle$ and its turbulent and wave parts are used in DLO0 and herein as proxies for the total wave energy, $E$, and for the TKE, $K$. The total energy density per unit mass of the internal wave field, $E$, is given by (Gill, 1982, p. 140)

$E=1 / 2\left[\left\langle\tilde{u}^{2}\right\rangle+\left\langle\tilde{v}^{2}\right\rangle+\left\langle\tilde{w}^{2}\right\rangle+N^{2}\left\langle\tilde{\zeta}^{2}\right\rangle\right]$.

Here, $\tilde{\zeta}$ is the wave-related vertical displacement of an isopycnal, and $\left\langle\tilde{u}^{2}\right\rangle$ and $\left\langle\tilde{v}^{2}\right\rangle$ are the horizontal wave velocity variances. In the sum of wave energy and TKE,

$\mathcal{E}=E+K$,

the relationship between $\mathcal{E}$ and $\left\langle w^{2}\right\rangle$ can be explored by writing

$\left\langle w^{2}\right\rangle=\left\langle\tilde{w}^{2}\right\rangle+\left\langle w^{\prime 2}\right\rangle \approx q \frac{E}{2}+\frac{2}{3} K$.

We assume isotropy for the turbulent part and equipartition between potential and kinetic energy for the wave part. The variable $q$ represents the ratio of vertical kinetic energy to $E$, $0<q<1$. DL00 show that $q$ is almost identical with the ratio $f / N$. Based on the GM reference value $N_{0}=5.2 \times 10^{-3} \mathrm{~s}^{-1}$, corresponding to a buoyancy period of about $20 \mathrm{~min}$, we conclude that $q$ can rarely exceed the value 0.03 . Further below we use $q=0.02$ for a latitude of $47^{\circ}$.

\subsection{The WT transition in our model: general solution}

We now examine how our model behaves as a function of varying internal wave and turbulent energy density. Commensurate with the idiosyncrasies of DL00, energy is largely discussed in terms of vertical velocity variance. 
For the wave-only case the general relationship Eq. (5) is transformed with Eq. (41) to

$\varepsilon=\pi^{-1} \Omega K=\pi^{-1} \eta K N \approx 0.34 K N$.

Further, we conclude from Eqs. (25) and (31) for the steady state, wave-only case that $\tilde{P} \approx \Pi \approx c_{2} E^{2}$, and with Eqs. (34 and 36) we obtain

$\varepsilon=c_{2}(1+\tilde{\Gamma})^{-1} E^{2}$.

Equating (51) and (52) produces

$K=\frac{\pi}{\eta} \frac{c_{2}}{1+\tilde{\Gamma}} \frac{E^{2}}{N} \approx 2.47 c_{2} \frac{E^{2}}{N}$,

which we insert in Eq. (50) to obtain

$\left\langle w^{2}\right\rangle \approx q \frac{E}{2}+1.65 c_{2} \frac{E^{2}}{N}$.

This equation is solved by

$E=\frac{q}{4} \frac{\sqrt{1+16 q^{-2}\left\langle w^{2}\right\rangle 1.65 c_{2} / N}-1}{1.65 c_{2} / N}$.

Inserting Eq. (55) into (52) results in a general relationship between $\varepsilon, N, q$, and $\left\langle w^{2}\right\rangle$. It contains no further unknowns:

$\varepsilon=0.83 c_{2}\left(\frac{q}{4} \frac{\sqrt{1+16 q^{-2}\left\langle w^{2}\right\rangle 1.65 c_{2} / N}-1}{1.65 c_{2} / N}\right)^{2}$.

\subsection{Asymptotically large and small energy density}

Before studying this general case we examine the ratio $\left\langle\tilde{w}^{2}\right\rangle /\left\langle w^{2}\right\rangle$, that is, the relative share of the waves of the total vertical velocity variance. We therefore take $\left\langle w^{2}\right\rangle$ from Eq. (54) and find

$\frac{q E}{2\left\langle w^{2}\right\rangle}=\frac{q N}{q N+1.65 c_{2} E}$.

We see that, when the wave energy level $E$ increases absolutely, it decreases relative to the total vertical velocity variance. In other words, the TKE share of $\left\langle w^{2}\right\rangle$ increases more strongly than $E$ and becomes dominating for very large $E$. This is independent of $q$. We reiterate that for the case under consideration large $E$ implies large $\Pi$ and large $\tilde{P}$.

The asymptotic cases of very small and very large energy densities and the associated energy flux $\Pi$ are especially interesting. In the asymptotic limit of $\Pi \rightarrow \infty$ we obtain $\left\langle w^{2}\right\rangle \rightarrow \frac{2}{3} K$, and, with Eq. (51),

$\varepsilon \approx 0.51 N\left\langle w^{2}\right\rangle$.

For very large total energy density and corresponding large energy flux into and out of the internal wave field the turbulent dissipation rate scales with the first power of the vertical velocity variance.

The opposite asymptotic case is that of vanishingly small energy flux, $\Pi \rightarrow 0$, such that also $E \rightarrow 0$. Due to Eq. (57)

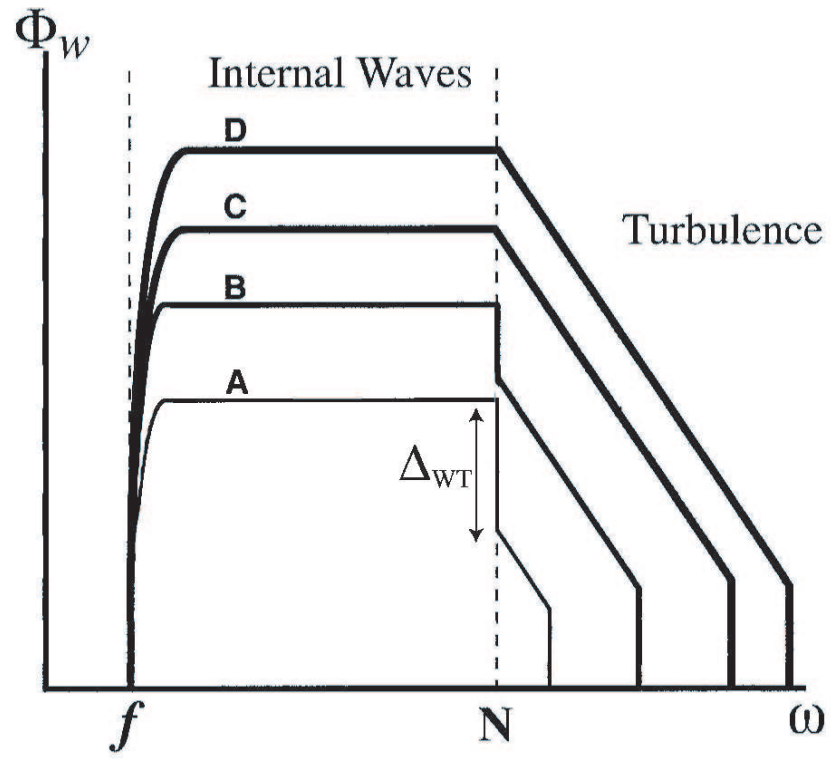

Fig. 2. Sketch of Lagrangian frequency spectra of vertical velocity from D'Asaro and Lien (2000; their Fig. 2), log-log plot. Waves span frequencies from $f$ to $N$ with $\omega^{0}$ behavior. Turbulence resides at $\omega>N$ with approximate $\omega^{-2}$ shape. With increasing energy, cases $A$ to $D$, the wave-turbulence transition is reached when the level of turbulence reaches that of the waves at $\omega=N, \Delta w_{T}=0$, case $\mathrm{C}$.

it follows that $\left\langle\tilde{w}^{2}\right\rangle /\left\langle w^{2}\right\rangle \rightarrow 1$. In other words we have $E \rightarrow 2\left\langle w^{2}\right\rangle / q$, which we insert into Eq. (52) to find

$\varepsilon=3.33 \frac{c_{2}}{q^{2}}\left\langle w^{2}\right\rangle^{2}$.

For very small total energy density and correspondingly small energy flux the turbulent dissipation rate scales with the second power of the vertical velocity variance. Our model thus qualitatively reproduces the energy and energy flux behavior of the WT transition as analyzed by DL00.

The transition from the low-energy to the high-energy state is continuous as shown in Fig. 3, which depicts the leading power $p$ of $\varepsilon$ in a power-law relationship with $\left\langle w^{2}\right\rangle$. The definition of $p$ is

$p=\frac{\left\langle w^{2}\right\rangle}{\varepsilon} \times \frac{d \varepsilon}{d\left\langle w^{2}\right\rangle}$,

where $\varepsilon$ has been calculated from the general relationship Eq. (56). Note how $p$ smoothly varies from 2 at small $\left\langle w^{2}\right\rangle$ to 1 at large $\left\langle w^{2}\right\rangle$. The dashed vertical line indicates the location of the WT transition threshold as discussed further below.

\subsection{The transition threshold}

As mentioned above and illustrated in Fig. 2, DL00 identify the threshold for the wave-turbulence regime transition with the lowest energy level at which $\Delta_{W T}=0$. In other words, 


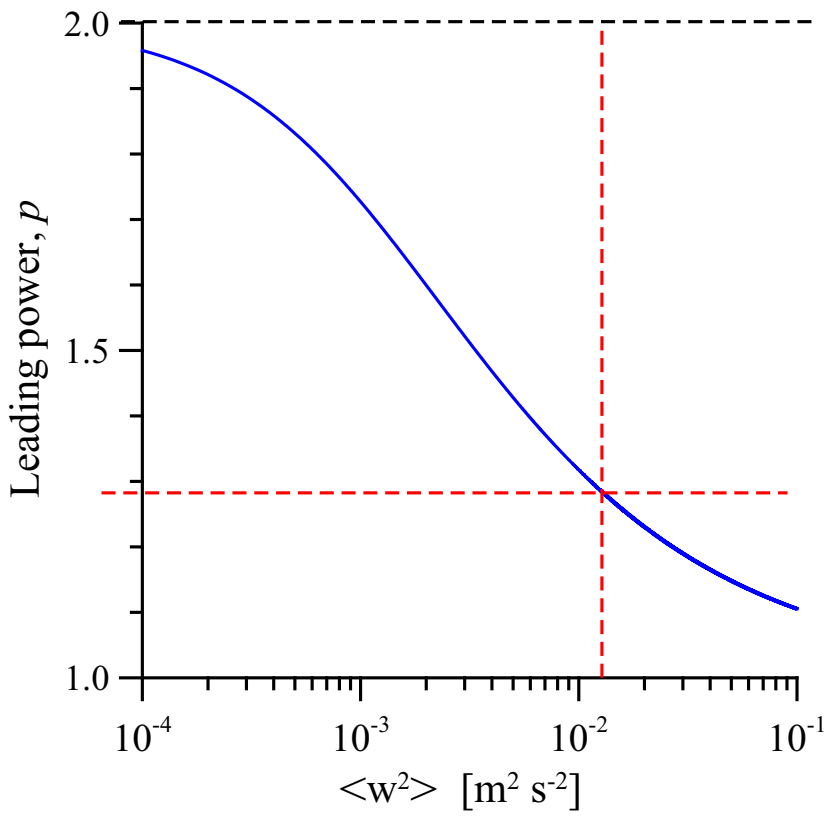

Fig. 3. Variation of the leading power $p$ of dissipation rate as function of the total vertical velocity variance of waves and turbulence combined, $\varepsilon \sim\left\langle w^{2}\right\rangle$. As the energy density of waves and turbulence increases the behavior of $\varepsilon$ changes from $\varepsilon \sim\left\langle w^{2}\right\rangle^{2}$ to $\varepsilon \sim\left\langle w^{2}\right\rangle^{1}$.

the transition is reached when $\Phi_{w}(\omega)$ becomes continuous at $\omega=N$. We exploit this condition in the following with some algebra.

The internal wave part of the total spectrum $\Phi_{w}, \Phi_{w}^{i w}$, is approximately white so that, with $f \ll N$, we have

$\left\langle\tilde{w}^{2}\right\rangle=\int_{f}^{N} \Phi_{w}(\omega) d \omega \approx N \times \Phi_{w}^{i w}$.

In the turbulent range, $\omega>N, \Phi_{w}(\omega)$ is approximately

$\Phi_{w}^{t}(\omega) \approx \beta \frac{\varepsilon}{\omega^{2}}$

following DL00. The Kolmogorov constant is $\beta \approx 1.8$. Upon integration of Eq. (46) the turbulent vertical velocity variance becomes

$\left\langle w^{\prime 2}\right\rangle \approx \beta \frac{\varepsilon}{N}$.

The threshold condition of DL00, $\Phi_{w}^{i w}(N)=\Phi_{w}^{t}(N)$, can be combined with Eqs. (61), (62) and (63) to obtain

$\left\langle w^{\prime 2}\right\rangle_{\mathrm{thr}} \approx\left\langle\tilde{w}^{2}\right\rangle_{\mathrm{thr}}$

Indices "thr" are used to indicate conditions valid only at the WT transition threshold. We further recall Eq. (50) and combine it with Eq. (63) and the threshold condition Eq. (64) as $\left\langle w^{2}\right\rangle_{\mathrm{thr}}=2 \beta \varepsilon_{\mathrm{thr}} / N$, or

$\varepsilon_{\mathrm{thr}}=(2 \beta)^{-1} N\left\langle w^{2}\right\rangle_{\mathrm{thr}} \approx 0.28 N\left\langle w^{2}\right\rangle_{\mathrm{thr}}$.

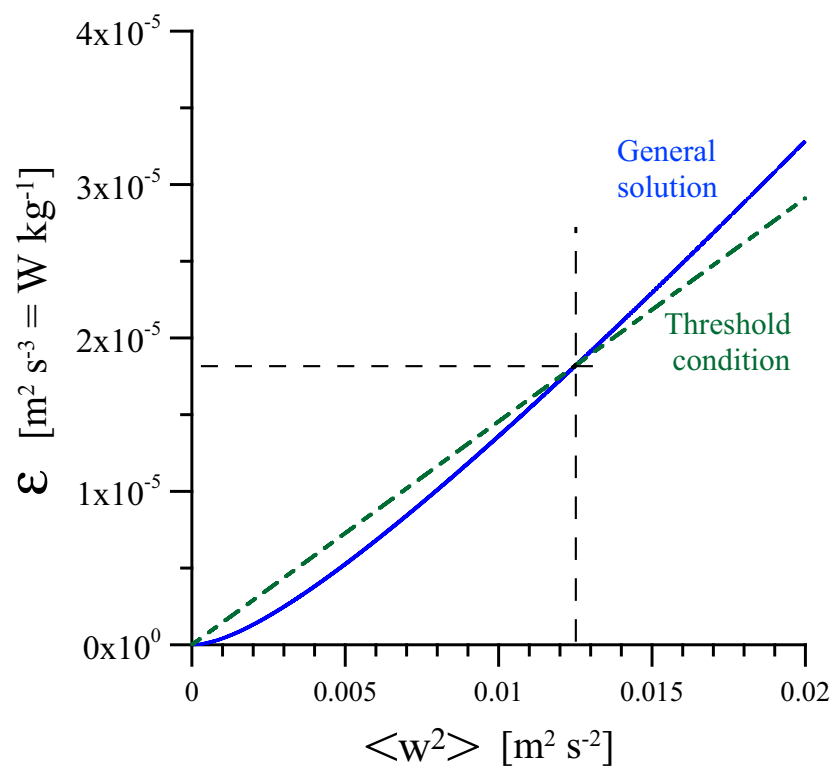

Fig. 4. Dissipation rate as a function of the vertical velocity variance in the general case Eq. (56) with $N=N_{0}=5.2 \times 10^{-3} \mathrm{~s}^{-1}$ and $q=0.02$. The coefficient $c_{2}=7.4 \times 10^{-5} \mathrm{~m}^{-2} \mathrm{~s}^{1}$ was chosen following G89. The dashed lines marks the location of the WT threshold for these values.

We can quantify $\varepsilon_{\text {thr }}$ and $\left\langle w^{2}\right\rangle_{\text {thr }}$ by combining Eq. (65) with the general solution Eq. (56),

$$
\begin{aligned}
& 0.28 N\left\langle w^{2}\right\rangle_{\mathrm{thr}}=0.83 c_{2}(q / 4)^{2} \times \\
& \left(\sqrt{1+16 q^{-2}\left\langle w^{2}\right\rangle_{\mathrm{thr}} 1.65 c_{2} / N}-1\right)^{2} \times \\
& \left(1.65 c_{2} / N\right)^{-2} .
\end{aligned}
$$

For $N=N_{0}$ the solution is $\varepsilon_{\text {thr }}=1.8 \times 10^{-5} \mathrm{~m}^{2} \mathrm{~s}^{-3}$ and $\left\langle w^{2}\right\rangle_{\mathrm{thr}}=0.0125 \mathrm{~m}^{2} \mathrm{~s}^{-1}$. This is consistent with DL00 who find $\varepsilon_{\text {thr }}=3 \times 10^{-5} \mathrm{~m}^{2} \mathrm{~s}^{-3}$ for a standard deep-water GM case. These results are depicted in Fig. 4, where the general solution Eq. (56) is plotted together with the threshold values $\varepsilon_{\text {thr }}$ and $\left\langle w^{2}\right\rangle_{\text {thr }}$. The asymptotic results Eqs. (58) and (59) can be summarized as follows, where $q=0.02$.

$\varepsilon=\left\{\begin{array}{l}0.62\left\langle w^{2}\right\rangle^{2}, E \ll E_{\mathrm{thr}}, \\ 0.51 N\left\langle w^{2}\right\rangle, E \gg E_{\mathrm{thr}} .\end{array}\right.$

According to Eq. (52), $E_{\mathrm{thr}}=\sqrt{(1+\tilde{\Gamma}) \varepsilon_{\mathrm{thr}} / c_{2}}$, which results in $E_{\text {thr }} \approx 0.54 \mathrm{~m}^{2} \mathrm{~s}^{-2}$ for $N=N_{0}$.

It may be of interest to note the value of the eddy diffusivity $\mu_{t}$ at the WT transition threshold. With the general $\mu_{t}=\tilde{\Gamma} \varepsilon N^{-2}$ and $N=N_{0}$ we find a threshold level of $\mu_{\mathrm{t}, \mathrm{thr}}=1.3 \times 10^{-1} \mathrm{~m}^{2} \mathrm{~s}^{-1}$, much larger than typical deep ocean diffusivities of the order of $10^{-5} \mathrm{~m}^{2} \mathrm{~s}^{-1}$ (G89).

\subsection{The high-energy regime}

The high-energy, linear behavior of $\varepsilon$ vs. $\left\langle w^{2}\right\rangle$ can be described following DL00 as $\varepsilon=C N\left\langle w^{2}\right\rangle$, where $C$ is expected 
to be a universal constant. DL00 find $C$ in the range of 0.3 to 0.6 and associate the uncertainty primarily with the uncertainty of Kolmogorov constant $\beta$. This can be compared with Weinstock (1981) who suggests $C$ in the range of 0.4 to 0.5 for stratospheric problems while Moum (1996b) finds $C=0.73 \pm 0.06$ in the oceanic thermocline. Our model yields $C=0.51$ as shown above and thus corresponds well to the results of DL00, Weinstock (1981) and Moum (1996b).

\section{Summary and conclusions}

In this paper, we develop a new two-equation model of stratified turbulence which covers the limits of shear-driven mixing without saturated waves, and internal wave-driven mixing without mean shear. The equation for TKE carries an extra source term, $\tilde{P}$, for the energy flux from saturated internal waves to turbulence,

$\frac{d K}{d t}=P+\tilde{P}-\mathcal{B}-\varepsilon$.

Saturated waves are defined herein as producing a significant $\tilde{P}$, which is often not the case under laboratory conditions. Equations $(4,11,12,13,14,15,32)$ specify the terms of Eq. (68). The equation for the turbulent enstrophy $\Omega$, does not carry a buoyancy-related term in the mean-shearonly case (i.e. $\tilde{P}=0$ ),

$$
\frac{d \Omega}{d t}=\frac{1}{\pi}\left(\frac{S^{2}}{2}-\Omega^{2}\right) .
$$

In the waves-only limit with vanishing mean shear, $S=0$, the enstrophy equation becomes

$$
\frac{d \Omega}{d t}=\frac{1}{\pi}\left(\eta^{2} N^{2}-\Omega^{2}\right) \text {, }
$$

where $\eta=1.06$ from Eq. (42).

The simple two-equation model of Eq. (68) combined with Eq. (70) in the waves-only, no mean shear case provides the turbulence with a frequency scale $\Omega \sim N$ and with a length scale $\sim L_{O}$ in agreement with oceanic observations. The model also exhibits the most important characteristics of the wave-turbulence transition as described by D'Asaro and Lien (2000b), $\varepsilon \sim E^{2}$ in the low-energy limit and $\varepsilon \sim E^{1}$ in the high energy limit. It is consistent with the quantitative location of the transition threshold in terms of $\varepsilon$ and vertical velocity variance in the deep ocean case. Our model is also quantitatively consistent with the behavior of the dissipation - energy relationship in the high-energy case, $\varepsilon=C\langle w\rangle^{2}$.

This and our previous studies present deliberately simple models aimed at physical and mathematical transparency and realism. They do not aim at optimally fitting model parameters. Thus, we do not find it worrisome, for example, that the neutral turbulent Prandtl number of our model, 0.5 following Eq. (15), is at low end of observed values. Because of the well known closure problem, all turbulence closures necessarily embody assumptions not directly based on first principles. Our novel model is no exception. We invite discussions of the physical and mathematical merits of our model including the assumptions on which it is based.

It is important to point out that we did not deduce our results from direct observations but rather derived them from a theory of stratified turbulence and internal waves which uses the asymptotic mixing efficiency $\tilde{\Gamma}=0.2$ and the Kolmogorov constant $\beta$ as the only empirical parameters. ${ }^{2}$ We had the intuition that Eq. (69) would retain its structure in the wavesonly case and then assumed that the mixing efficiency would stay finite and constant in the limit of vanishing mean shear. These two assumptions proved to be sufficient to work out our new model.

In producing the correct space and time scales for oceanic wave-driven mixing and in replicating the wave-turbulence transition of DL00 our model appears promising. Naturally, it has limitations, too, limitations beyond treating only the limits of no mean shear and no saturated waves. In the case of $S=0$ it addresses the generation of mixing by a deep-ocean, GM-like internal wave field. Other cases, such as mixing on the comparatively shallow continental shelf are beyond its scope for now. In order to make the new model useful in a practical, rather than theoretical, sense, the coexistence of waves and mean shear has to be allowed for. Work on this difficult scenario has begun.

Acknowledgements. This study was supported in part by the United States National Science Foundation (grant OCE-0352047), by ONR-Global and by a grant from the Deutsche Forschungsgemeinschaft. We thank Eric D'Asaro for enlightening discussions, critical commentary, encouragement to follow our path, and the kind permission to use sketches from DL00. HZB thanks Rupert Klein for his vigorous support and interesting insights into vortex theory. Comments by two anonymous reviewers helped us improve the manuscript.

Edited by: J. Schröter

\section{References}

Abarbanel, H. D. I., Holm, D. D., Marsden, J. E., and Ratio, T.: Richardson number criterion for the nonlinear stability of threedimensional stratified flow, Phys. Rev. Lett., 26, 2352-2355, 1984.

Baumert, H.: A Novel Two-Equation Turbulence Closure for high Reynolds numbers. Part B: Spatially Non-Uniform Conditions, in: Marine Turbulence, Theories, Observations and Models, edited by Baumert, H., Simpson, J., and Sündermann, J., Cambridge University Press, Cambridge, UK, 4, 31-43, 2005.

Baumert, H. and Peters, H.: Second Moment Closures and Length Scales for Stratified Turbulent Shear Flows, J. Geophys. Res., 105, 6453-6468, 2000.

Baumert, H. and Peters, H.: Turbulence Closure, Steady State, and Collapse into Waves, J. Phys. Oceanogr., 34, 505-512, 2004.

\footnotetext{
${ }^{2}$ The external variable $q \approx f / N$ does not belong to the class of model parameters.
} 
Baumert, H. and Peters, H.: A Novel Two-Equation Turbulence Closure for high Reynolds numbers. Part A: Homogeneous, NonRotating Stratified Shear Layers, in: Marine Turbulence, Theories, Observations and Models, edited by Baumert, H., Simpson, J., and Sündermann, J., Cambridge University Press, Cambridge, UK, 14-30, 2005.

Canuto, V. M., Howard, A., Cheng, Y., and Dubovikov, M. S.: Ocean turbulence I: One-point closure model. Momentum and heat vertical diffusivities, J. Phys. Oceanogr., 31, 1413-1426, 2001.

Crawford, W. R.: A Comparison of Length Scales and Decay Times of Turbulence in Stratified Flows, J. Phys. Oceanogr., 16, 18471854, 1986.

D'Asaro, E. A. and Lien, R. C.: Lagrangian measurements of waves and turbulence in stratifed flows, J. Phys. Oceanogr., 30, 641655, 2000a.

D'Asaro, E. A. and Lien, R. C.: The Wave-Turbulence Transition for Stratified Flows, J. Phys. Oceanogr., 30, 1669-1678, $2000 \mathrm{~b}$.

Dickey, T. D. and Mellor, G. L.: Decaying Turbulence in Neutral and Stratified Fluids, J. Fluid Mech., 99, 37-48, 1980.

Dillon, T. M.: Vertical Overturns: A Comparison of Thorpe and Ozmidov Length Scales, J. Geophys. Res., 87, 9601-9613, 1982.

Foken, T.: 50 years of the Monin-Obukhov similarity theory, Bound.-Lay. Meteorol., 119, 431-447, 2006.

Fritts, D. and Alexander, J.: Middle atmosphere gravity wave dynamics, Rev. Geophys., 41, 1-64, 2003.

Fritts, D. C.: A review of gravity wave saturation processes, effects and variability in the middle atmosphere, Pure Appl. Geophys., 130, 242-371, 1989.

Galperin, B., Sukoriansky, S., and Anderson, P. S.: On the critical Richardson number in stably stratified turbulence, Atmos. Sci. Lett., 8, 65-69, 2007.

Garrett, C. J. R. and Munk, W. H.: Space-Time Scales of Internal Waves, Geophys. Astrophys. Fluid Dyn., 3, 225-264, 1972.

Garrett, C. J. R. and Munk, W. H.: Space-Time Scales of Internal Waves: A Progress Report, J. Geophys. Res., 80, 291-297, 1975.

Gill, A. E.: Atmosphere-Ocean Dynamics, Academic Press, New York, 662 pp., 1982.

Gregg, M. C.: Scaling of Turbulent Dissipation in the Thermocline, J. Geophys. Res., 94, 9686-9697, 1989.

Gregg, M. C., Winkel, D. P., and Sanford, T. B.: Varieties of Fully Resolved Spectra of Vertical Shear, J. Phys. Oceanogr., 23, 124$141,1993$.

Henyey, F. S., Wright, J., and Flatté, S. M.: Energy and Action Flow Through the Internal Wave Field: An Eikonal Approach, J. Geophys. Res., 91, 8487-8495, 1986.

Howard, L. N.: Notes on a paper of John W. Miles, J. Fluid. Mech., 10, 509-512, 1961.

Li, M., Zhong, L., and Boicourt, W. C.: Simulations of Chesapeake Bay estuary: Sensitivity to turbulence mixing parameterizations and comparison with observations, J. Geophys. Res.Oceans, 110, C12004, doi:10.1029/2004JC002585, 2005.

MacKinnon, J. A. and Gregg, M. C.: Mixing on the Late-Summer New England Shelf - Solibores, Shear, and Stratification, J. Phys. Oceanogr., 33, 1476-1492, 2003.

McComas, C. H. and Müller, P.: The Dynamic Balance of Internal Waves, J. Phys. Oceanogr., 11, 970-986, 1981.

Mellor, G. L. and Yamada, T.: A Hierarchy of Turbulence Closure models for Planetary Boundary Layers, J. Atmos. Sci., 31, 1791-
1806, 1974.

Mellor, G. L. and Yamada, T.: Development of a Turbulence Closure Model for Geophysical Fluid Problems, Rev. Geophys., 20(4), 851-875, 1982.

Miles, J. W.: On the stability of heterogeneous shear flow, J. Fluid. Mech., 10, 496-508, 1961.

Moum, J. N.: The Quest for $K_{\rho}$ - Prelimiary Results From Direct Measurements of Turbulent Fluxes in the Ocean, J. Phys. Oceanogr., 20, 1980-1984, 1990.

Moum, J. N.: Efficiency of Mixing in the Main Thermocline, J. Geophys. Res., 101, 12 057-12 070, 1996 a.

Moum, J. N.: Energy-Containing Scales of Stratified Turbulence, J. Geophys. Res., 101, 14 095-14 109, 1996b.

Oakey, N. S.: Determination of the Rate of Dissipation of Turbulent Energy From Simultaneous Temperature and Velocity Shear Microstructure Measurements, J. Phys. Oceanogr., 12, 256-271, 1982.

Osborn, T. R.: Estimates of the Local Rate of Vertical Diffusion from Dissipation Measurements, J. Phys. Oceanogr., 10, 83-89, 1980.

Peters, H. and Baumert, H. Z.: Validating a Turbulence Closure Against Estuarine Microstructure Measurements, Ocean Modelling, 19, 183-203, 2007.

Polzin, K., Toole, J. M., and Schmitt, R. W.: Finescale Parameterizations of Turbulent Dissipation, J. Phys. Oceanogr., 25, 306328, 1995.

Richardson, L. F.: Weather prediction by numerical processes, Cambridge Univ. P., London, 236 pp., 1922.

Rodi, W.: Examples of Calculation Methods for Flow and Mixing in Stratified Fluids, J. Geophys. Res., 92, 5305-5328, 1987.

Rohr, J. J., Itsweire, E. C., Helland, K. N., and Van Atta, C. W.: Growth and Decay of Turbulence in a Stably Stratified Shear Flow, J. Fluid Mech., 195, 77-111, 1988.

Schumann, U. and Gerz, T.: Turbulent Mixing in Stably Stratified Shear Flows, J. Appl. Meteorol., 34, 33-48, 1995.

Shih, L. H., Koseff, J. R., Ferziger, J. H., and Rehmann, C. R.: Scaling and Parameterization of Stratified Homogeneous Turbulent Shear Flow, J. Fluid Mech., 412, 1-20, 2000.

Sukoriansky, S.: On the critical Richardson number in stably stratified turbulence, Atmospheric Science Letters, 8(3), 65-69, 2007.

Sukoriansky, S., Galperin, B., and Perov, V.: A quasi-normal scale elimination model of turbulence and its application to stably stratified flows, Nonlinear. Proc. Geoph., 13, 9-22, 2006.

Tjernström, M.: Turbulence length scales in stably stratied flows analyzed from slant aircraft proiles, J. Climate, 32, 948-963, 1993.

Weinstock, J.: Vertical Turbulence Diffusivity for Weak and Strong Stable Stratification, J. Geophys. Res., 86, 9925-9928, 1981.

Wijesekera, H. W. and Dillon, T. M.: Shannon Entropy as an Indicator of Age for Turbulent Overturns in the Oceanic Thermocline, J. Geophys. Res., 102, 3279-3291, 1997.

Wilcox, D. C.: Turbulence modeling for CFD, DCW Industries, Inc., La Canada, CA, 2nd edn., 540 pp., 1998.

Zilitinkevich, S. S., Elperin, T., Kleeorin, N., and Rogachevskii, I.: Energy- and flux-budget (EFB) turbulence closure model for the stably stratified flows. Part I: Steady-state, homogeneous regimes, Bound.-Lay. Meteorol., 125, 167-191, 2007. 